\title{
Study of Periodic Fabrication Error of Optical Splitter Device Performance
}

\author{
Mohammad Syuhaimi Ab-Rahman ${ }^{\#}$, Foze Saleh Ater ${ }^{\#}$, Kasmiran Jumar ${ }^{\text {i\# }}$, Rahmah Mohammad \\ ${ }^{*}$ Spectrum technology Research Division (SPECTEC) \\ Faculty of Engineering \& Built Environment, Department of Electrical, Electronic and Systems Engineering \\ Universiti Kebangsaan Malaysia (UKM), 43600 Bangi, Selangor Malaysia \\ E-mail : syuhaim@vlsi.eng.ukm,aterfoze@yahoo.com \\ * Universiti Teknologi Mara (UiTM) \\ E-mail :rahmah@technologist.com
}

\begin{abstract}
In this paper, the effect of fabrication errors (FEs) on the performance of $1 \times 4$ optical power splitter is investigated in details. The FE, which is assumed to take regular shape, is considered in each section of the device. Simulation result show that FE has a significant effect on the output power especially when it occurs in coupling regions.
\end{abstract}

Keywords - Optical power splitter, Output power, Fabrication error, Simulation.

\section{INTRODUCTION}

Optical power splitter is widely used as power divider or combiner in modulator, switches, interferometer devices and semiconductor lasers [1-3]. Its main function is to split the incoming light into multi paths where each path may carry same or different ratio of power. These devices can be considered as the best and simple approach to split light in a planar lightwave circuit [10].

In addition, optical splitter devices are important components for photonic and optoelectronic integrated circuits due to their simple structure, low loss, and large optical bandwidth. They play a central role in passive optical distribution networks [1,2]. Optical power splitter should meet practical requirements such as type of material, dimensions and the accuracy of fabrication process. In addition, they have attracted considerable interests due to advantageous characteristics such as compactness, relaxed large bandwidth and fabrication tolerance [4-9].

Fabrication processes, such as spin coating is still attracted due low cost. However, the accuracy of this method in term of dimensions of the produced device is not high. This would lead to re-fabricate the same sample many times until the achievement of exact design. In this paper, the effect of less accuracy in fabrication process, which is represented as fabrication error, on the output power for $1 \times 4$ optical splitter is investigated details. In other words, the fabrication error is considered so that the width of waveguide is deviated in specified sections. This deviation is assumed to take a periodic shape, instead of random one (which occurs in reality) for the sake the simplicity [10].

\section{DESIGN}

The Schematic view of the designed $1 \times 4$ optical power Splitter is shown in Fig. 1 with labeled sections. The basic unit of device, $1 \times 2 \mathrm{Y}$ - branch section, consists of linear waveguide, and a pair of S-bend sine branching wave guide [8]. The dimensions of the layout wafer are $1200 \mu \mathrm{m}$ (length) $\times 65 \mu \mathrm{m}$ (width). The refractive indices, of the core and cladding, which are used in simulation, are 1.5 and 1.35 respectively at wavelength of $1.55 \mu \mathrm{m}$. The dimensions of whole structure are $1200 \mu \mathrm{m}$ (total length) $\times 6 \mu \mathrm{m}$ (width of waveguide). Due to consideration of error in fabrication in this paper, the width of waveguide is assumed to be changed periodically in a specified section as shown in Fig. 2. This figure shows the schematic view of error samples with the corresponding dimensions. The error section shown in Fig. 2 is considered at different positions of structure. The dimensions and the shape of each sample error are chosen according to the nearest shape of real FE which takes random shape. The representation of FE in a shape, such that the width of waveguide in a certain section is changed periodically as shown in fig. 2, enables the designer to investigate the effect of error on the output power of optical 
splitter. In addition, consideration of FEs at different sections will give us technical information about whether its effect will degrade the performance of splitter or not. Thus, if the effect of FE, in a specified section, on the output power is small, the corresponding section can be cut or removed without significant degradation in output power. This will give flexibility in fabrication and the corresponding cost. However, when the FE occurs in branching region, i.e. S-bend sections, the output will be degraded significantly as will be shown later in simulation results, therefore, the treatment may requires the refabrication of the whole device.

\section{RESULTS AND DISCUSSION}

The simulation results are performed using beam propagation method (BPM) software tool [11]. Firstly, figure 3 shows the output power for each port of $1 \times 4$ optical splitter assuming errorless in fabrication process. As shown in this figure, the output power is divided equally for each two port, namely the inner and the outer branches. The percentage of normalized total output power is $97.924 \%$ as shown in Fig. 13. The values of core and cladding refractive indices and the width of waveguide have been optimized to achieve this value of output power.

Figures 4 through 12 show the layout and the corresponding output power (for each port) when considering the FE in section A through I respectively. As shown in Fig. 4, where the $\mathrm{FE}$ is considered in section $\mathrm{A}$, the total output power is reduced to $83.251 \%$ as shown in Fig. 13. In addition, it can be observed in Fig. 4 that the output power is higher in port 2 compared with others due to changes in optical field propagation.

When considering of the FE in section B as shown in Fig. 5 (see also Fig. 13 for total output power), the output power is reduced in ports 1,2 and 4 while it increases in port 3 due to the existence the error in the coupling region. This also occurs when considering the $\mathrm{FE}$ in section $\mathrm{C}$ as shown in Fig. 6. It can be noted that the power decreases significantly in Ports 3 and 4. Thus, this indicates that the performance of the device will be degraded when the FE occurs in S-bend sections around the branching point. When the FE is considered in sections $\mathrm{D}$ and $\mathrm{E}$ as shown in Figs. 7 and 8, the output power in corresponding ports will decrease relatively. The corresponding total output power decreases compared with the case of no FE as shown in Fig. 13.

Moreover, When the FE is considered in the output Sbend waveguides, namely sections $\mathrm{E}$ through I as shown in Figs. 9 through 12 respectively, the output power will be affected directly in the corresponding port. It can be deduced with help of Fig. 13 that:

(i). The FE is more affected when it occurs in branching waveguide, i.e. S-bend sections. Technically, the treatment for this kind of error is difficult to be treated.

(ii). The FE is affected when it occurs in the main linear waveguide, i.e. section A but it can be treated by removing/cutting the degraded part without affecting significantly the performance of device. This may improve the total output power due to the reduction in length where the attenuation loss will be decreased accordingly. (iii). This also can be done when the FE is occurred in output branches. However, the integration/coupling with the commercially available $\mathrm{V}$-groove fiber array ribbon should be taken into account.

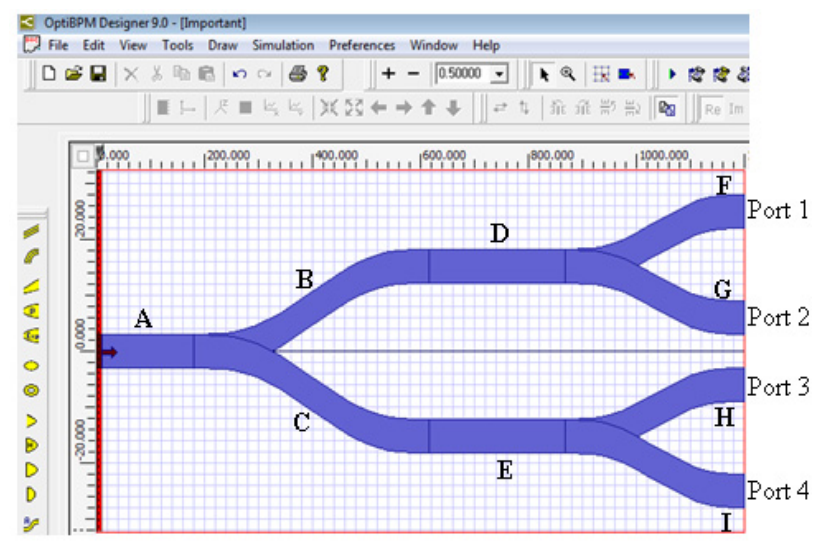

Fig. 1 Schematic view of $1 \times 4$ optical power Splitter

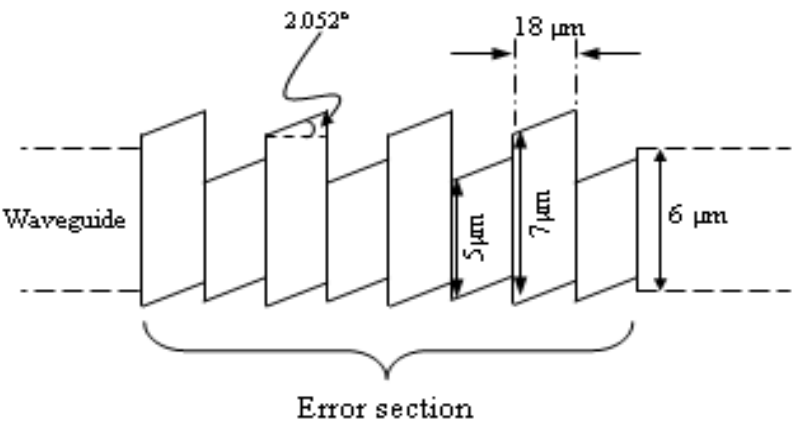

Fig. 2 Schematic representation of error section

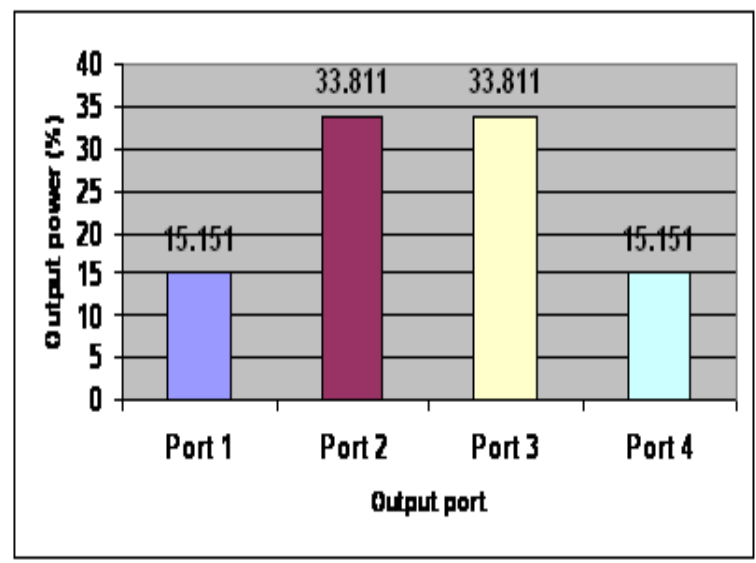

Fig. 3 The output power in each output port for the optical device assuming errorless in fabrication process 


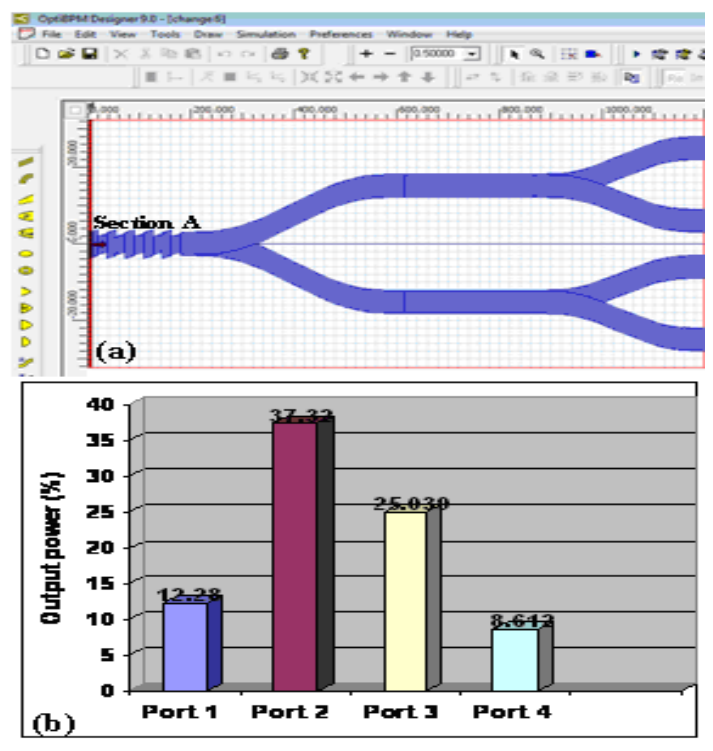

Fig. 4 (a) Schematic device layout considering the fabrication error in section A, (b) The corresponding output power in each port
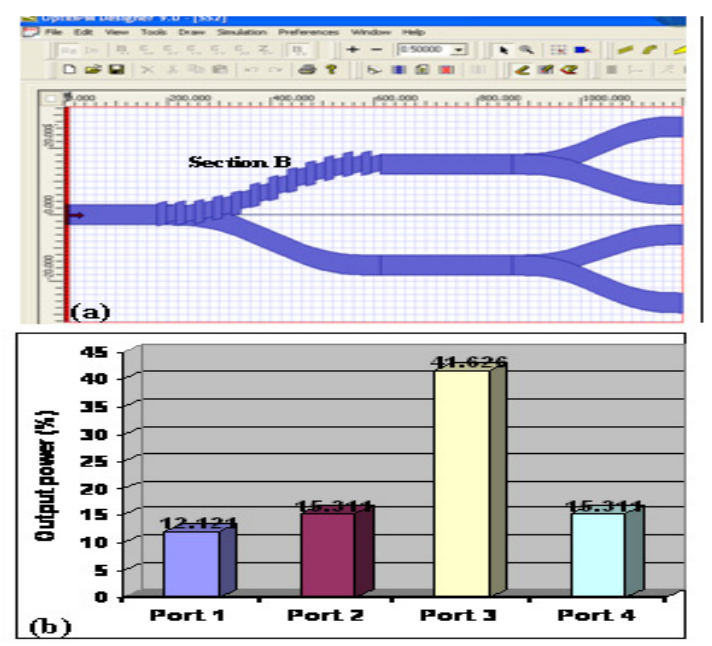

Fig. 5 Is the same as figure 4 but the fabrication error is considered in section B
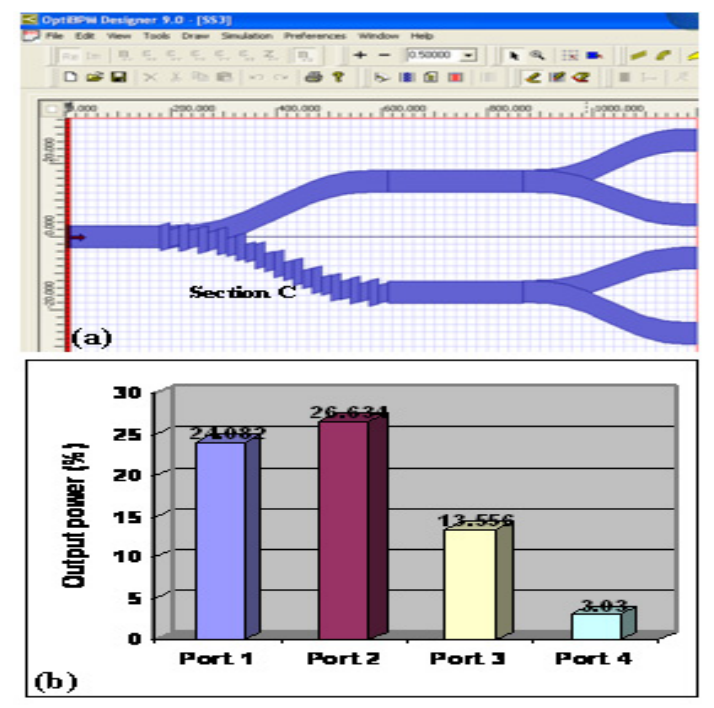

Fig. 6 Is the same as figure 4 but the fabrication error is considered in section $\mathrm{C}$

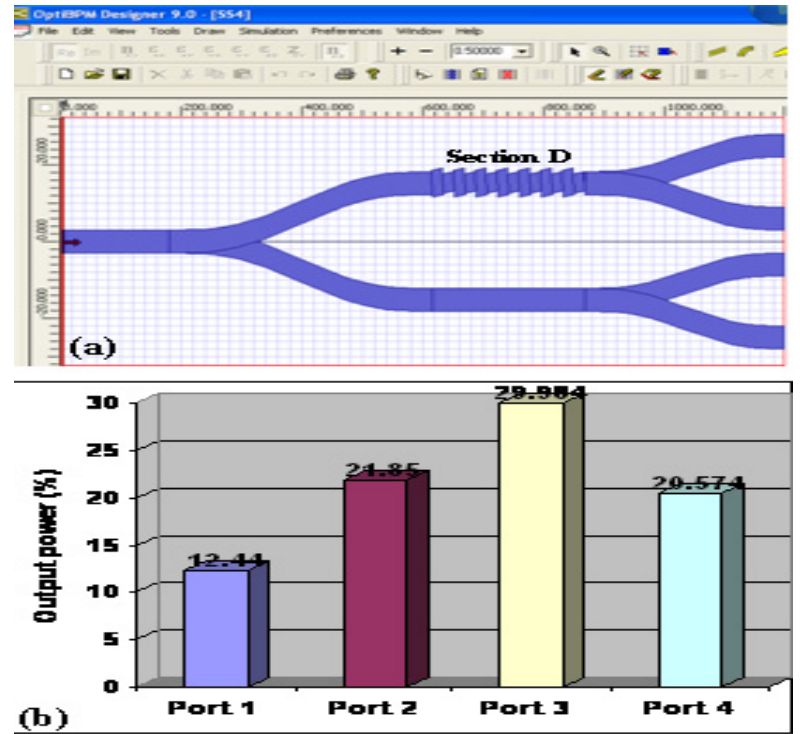

Fig. 7 Is the same as figure 4 but the fabrication error is considered in section D.

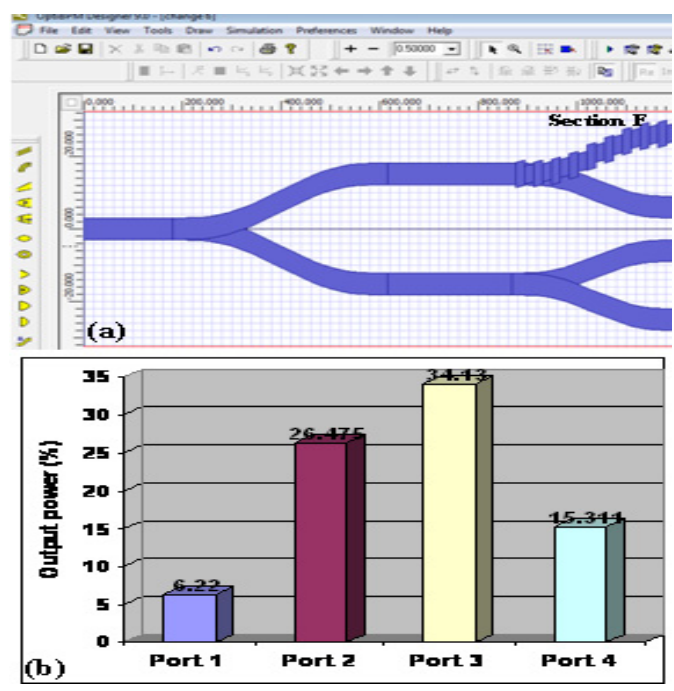

Figure 9: is the same as figure 4 but the fabrication error is considered in section $\mathrm{F}$.
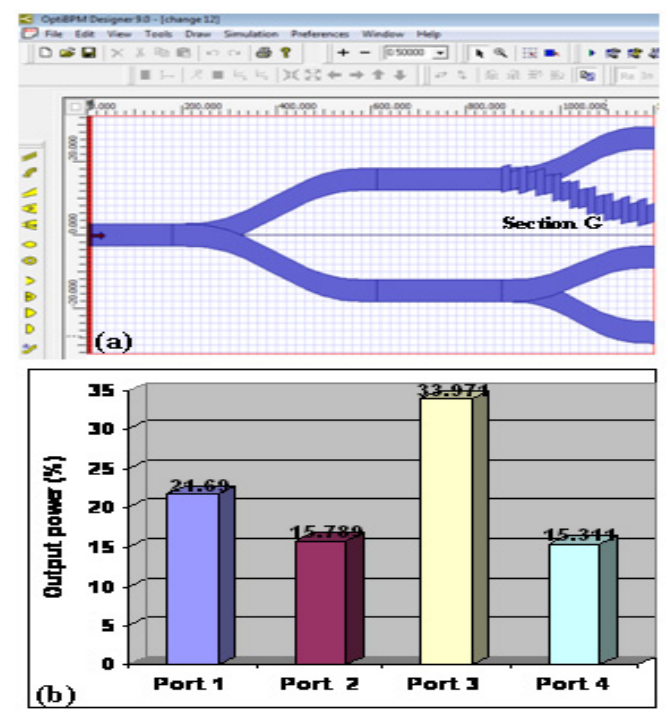

Fig. 10 Is the same as figure 4 but the fabrication error is considered in section $\mathrm{G}$ 


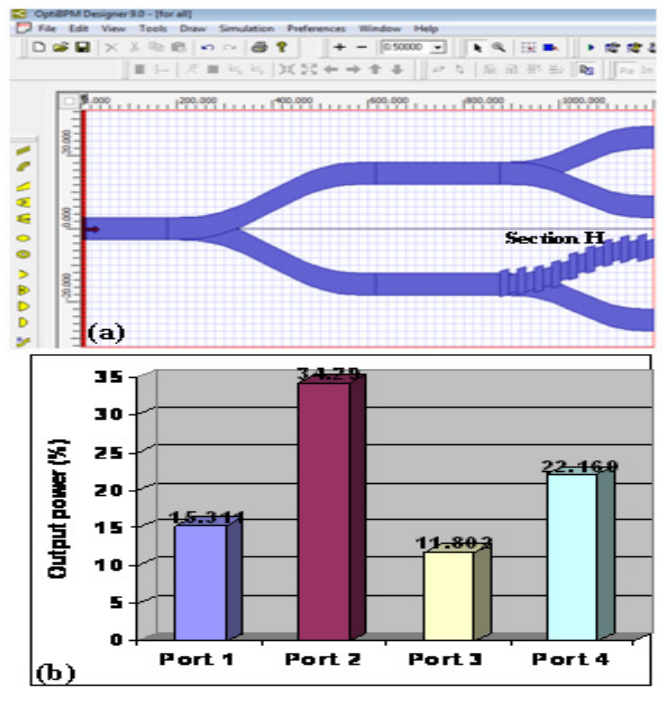

Fig. 11 Is the same as figure 4 but the fabrication error is considered in section $\mathrm{H}$

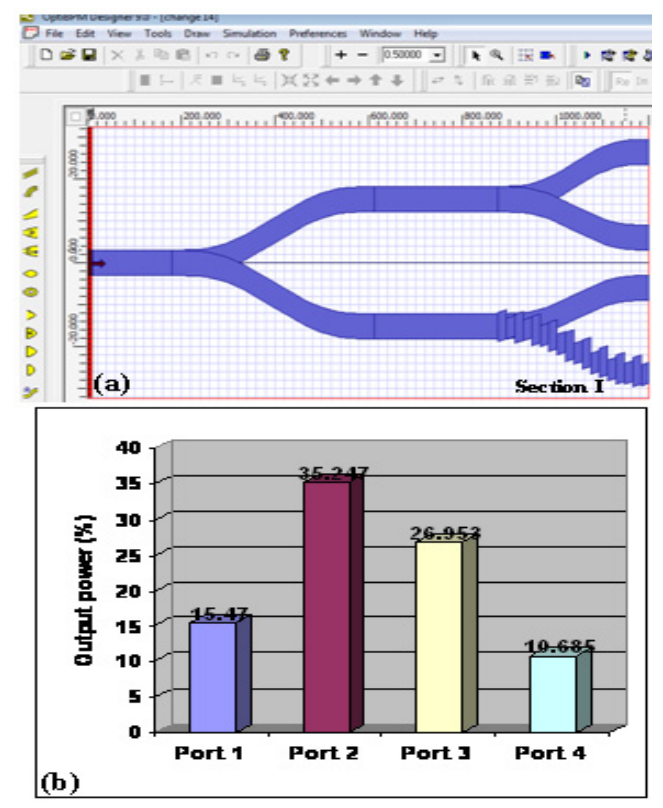

Fig. 12 Is the same as figure 4 but the fabrication error is considered in section I

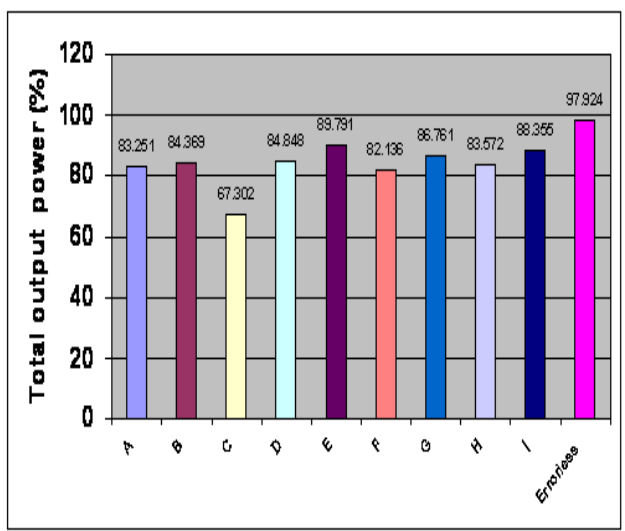

Fig. 13 Total output power of $1 \times 4$ optical power splitter considering fabrication errors in each section.

\section{CONCLUSIONS}

The effect of FE on the performance $1 \times 4$ optical power splitter is investigated in details. The FE has bee considered in each section of the device. Simulation results performed by BPM software have shown the FE is most affected when it occurs in the main linear section (where the whole total output power is reduced) and the branching sections (where the output power in the corresponding port will be affected directly) [11]. The benefit of this study is that the designer can predict the impact of FE previously when considering a specified design in fabrication. Moreover, even when employing low cost method such as spin coating (where the dimensions of the device may deviate from the optimized design due to less accuracy), the produced device may still acceptable due to less effect on the output power or due to easy treatment to the degraded section (error section).

\section{ACKNOWLEDGMENT}

This work is sponsored by Universiti Kebangsaan Malaysia (UKM) through UKM-AP-ICT-17-2009

\section{REFERENCES}

[1] H. Hatami-Hanza, P.L. Chu and M.J. Lederer, "A new low-loss wide-angle Y-branch configuration for optical dielectric slab waveguide", IEEE Photonics Technology Letters, vol. 6, pp. 528530, April 1994.

[2] Zainuddin L., Imran A., Ary S., Azliza J.M., and Chan W. Y.,"Analysis of Y-junction waveguide using the method of line", IEEE, June 2009.

[3] Y. Qian, J. Song, S, Kim, W. Hu, and Gregory P. Nordin, "Compact waveguide splitter networks", Electrical and Computer Engineering, 2008.

[4] A. Baby, C. Dhanavantri, J. Pachauri, S. Johri, P. Kumar and B. Singh, "Silica-on-silicon based $1 \times \mathrm{N}$ optical splitter: Design, fabrication and characterization" Indian Journal of Engineering \& Materials Sciences vol. 12, pp. 12-16, February 2005.

[5] C. Gi Choi, S. Han, B. Cheol Kim, S. Ahn, and M. Jeong, "Fabrication of large-core $1 \times 16$ optical power splitter in polymers using hot-embossing process", IEEE Photonics Technology Letters, vol. 15, June 2003.

[6] M. H. Ibrahim, N. M. Kassim, A. B. Mohammad, M. K. Chin, S. Y. Lee "Polymeric optical splitter based on multimode interference mechanism", IEEE, June 2006.

[7] Y. Cai, T. Mizumoto, and Y. Naito, “ A novel $1 \times 8$ optical power splitter using tapered waveguide coupling”, IEEE Photonics Technology Letters, vol. 3, February 1991.

[8] T. Yabu, M. Geshiro, and M. Ohashi, “ Novel design method for 4branch optical power splitters" Australian Conference on Optical Fiber Technology, July 2008.

[9] Kerstin W. Paul V. Lambeck, and A. Driessen, "Design, tolerance analysis, and fabrication of silicon oxynitride based planar optical waveguides for communication devices", IEEE Lightwave Technology, vol. 17, No.8, August 1999

[10] Jun S. and Ning Z., "Analysis method of the effect of fabrication on a planar waveguide demultiplexer", Optical and Quantum Electronics, 30 March 2006

[11] OptiBPM, Waveguide design software, version 9 